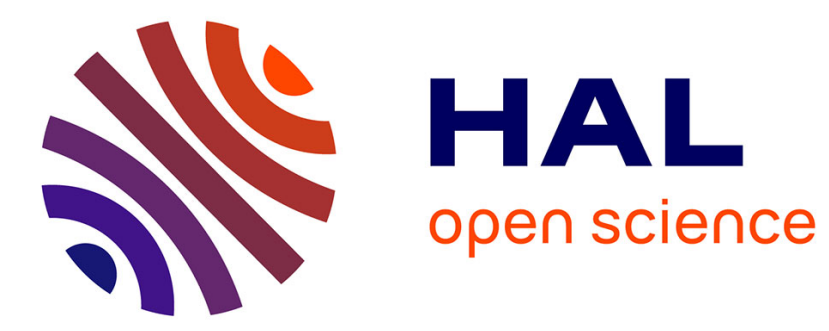

\title{
Far beyond primary poly(vinylamine)s through free radical copolymerization and amide hydrolysis
}

Mathilde Dréan, Philippe Guégan, Christine Jérôme, Jutta Rieger, Antoine Debuigne

\section{- To cite this version:}

Mathilde Dréan, Philippe Guégan, Christine Jérôme, Jutta Rieger, Antoine Debuigne. Far beyond primary poly(vinylamine)s through free radical copolymerization and amide hydrolysis. Polymer Chemistry, 2015, 7, pp.69-78 10.1039/C5PY01325A . hal-01221255

\section{HAL Id: hal-01221255 https: / hal.sorbonne-universite.fr/hal-01221255}

Submitted on 27 Oct 2015

HAL is a multi-disciplinary open access archive for the deposit and dissemination of scientific research documents, whether they are published or not. The documents may come from teaching and research institutions in France or abroad, or from public or private research centers.
L'archive ouverte pluridisciplinaire $\mathbf{H A L}$, est destinée au dépôt et à la diffusion de documents scientifiques de niveau recherche, publiés ou non, émanant des établissements d'enseignement et de recherche français ou étrangers, des laboratoires publics ou privés. 


\title{
Far Beyond Primary Poly(vinylamine)s Through Free Radical Copolymerization and Amides Hydrolysis
}

Cite this: DOI: 10.1039/C5Pyo1325a

Received 20th August 2015

Accepted 5th October 2015

DOI: $10.1039 /$ c5pyo1325a

\author{
Mathilde Dréan, ${ }^{\mathrm{a}, \mathrm{b}}$ Philippe Guégan, ${ }^{\mathrm{b}}$ Christine Jérôme, ${ }^{\mathrm{a}}$ Jutta Rieger, ${ }^{\mathrm{b} *}$ \\ Antoine Debuigne ${ }^{\mathrm{a} *}$
}

Due to their affinity for many supports, their $\mathrm{pH}$ responsiveness, metal binding capacity and polyelectrolytes complexation, poly(vinylamine) derivatives have attracted attention for many applications including coatings, water purification, or gas membrane separation. Nevertheless, most of them possess only pendant primary amines despite the possible benefits of incorporating different amino groups along the chain. In this work, a straightforward and scalable synthesis route towards polymers bearing primary and secondary amines, as well as imidazole groups, is reported. The general strategy relies on the radical copolymerization of different vinylamides and vinyl imidazole followed by the hydrolysis of the resulting poly(vinylamide) derivatives. Binary and ternary free radical copolymerizations of $\mathrm{N}$ vinylacetamide (NVA), N-methyl vinylacetamide (NMVA) and 1-vinylimidazole (VIm) were investigated and the reactivity ratios for each copolymerization system were determined. Thanks to these values a series of statistical copolymers with predictable composition and low deviation over the chain distribution could then be synthesized. Finally, the acidic hydrolysis of the acetamide functions towards the corresponding amine was performed and optimized. Copolymers containing various pendant amino groups and with low dispersity in the chain composition could be obtained, which opens new perspectives for the above mentioned applications.

\section{Introduction}

Poly(vinylamine) (PVAm) is a special and highly valuable macromolecule because it exhibits the highest possible density of pendant primary amines among all vinyl polymers. ${ }^{1}$ Excellent water solubility, metal binding capacity, ${ }^{2,3}$ ionization/pH behavior, ${ }^{4}$ polyelectrolyte complexation, ${ }^{5}$ and affinity for many supports ${ }^{6,7}$ are key properties of PVAm that make it useful for a broad range of applications, such as water purification, ${ }^{8}$ paper additive and coating applications, ${ }^{9-11}$ gas membrane separation, ${ }^{12,13}$ or functionalization of macroscopic and nanoparticle surfaces. ${ }^{6}$

The huge potential of this polymer has led to intensive research for the development of straightforward synthetic routes towards PVAm and derivatives. ${ }^{1}$ Because PVAm cannot be prepared from vinylamine due to its low stability and fast conversion into the corresponding imine through tautomeric equilibrium, ${ }^{14}$ multistep procedures had to be developed (Scheme 1). The Hoffman rearrangement of a polyacrylamide precursor ${ }^{15}$ successfully yielded polymers with a high density of pendant primary amines but this approach suffers from producing non-negligible amounts of side products, such as carboxyl and urea groups along the chain. ${ }^{16}$ In this respect, the radical polymerization of $\mathrm{N}$-vinylamides, mainly $\mathrm{N}$ vinylformamide (NVF) $)^{17-19}$ or $N$-vinylacetamide (NVA), ${ }^{20,21}$ with subsequent hydrolysis of the amides groups is "cleaner" and more straightforward. Optimal conversion of PNVF into PVAm can be achieved upon basic treatment, ${ }^{17,18}$ whereas high level of hydrolysis of the amide functions of PNVA was reached under acidic conditions. $^{20}$ Except for the cost of the monomer $(N-$ vinylphtalimide), hydrazinolysis of poly $(N$-vinylphtalimide) (NVPI) is another interesting route towards PVAm. ${ }^{22}$
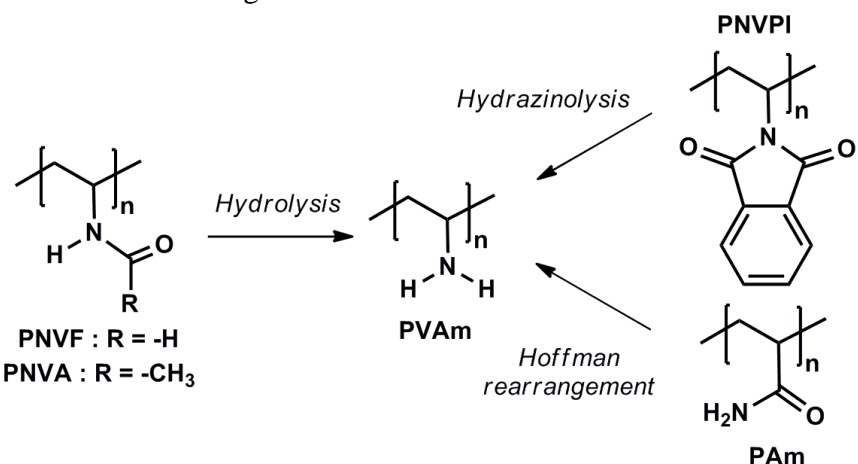

Scheme 1. Synthetic routes towards poly(vinylamine).

In order to meet the needs of today's applications, it is crucial to adjust the properties of PVAm and to provide it with additional functionalities by incorporating supplementary chemical moieties in its backbone. So far, the functionalization of PVAm is largely dominated by post-polymerization derivatization strategies arising from the ease of coupling amines with reactive molecules. ${ }^{1}$ In this way, several chemical groups have been successfully incorporated by post-treatment of PVAm including fluorinated ${ }^{23}$ and other hydrophobic groups, ${ }^{24}$ sugars,${ }^{25,26}$ antibodies, ${ }^{27}$ dyes, ${ }^{28}$ nucleic 
acids. $^{29}$ Another strategy for derivatizing PVAm consists in the radical copolymerization of vinylamides with selected comonomers followed by the hydrolysis of the amides. For example, NVF was copolymerized with vinylesters, acrylates or acrylamides, followed by acid or basic treatment leading to the corresponding polyvinylamine-based copolymers. ${ }^{30,31}$ Although straightforward, this approach requires a perfect knowledge of the comonomer reactivity ratios and close examination of the fate of the comonomer units during hydrolysis. Nevertheless, despite the possible benefits of incorporating various amino groups along the polymer chain ${ }^{32,33}$ poly(vinylamine)-based copolymers are generally restricted to pendant primary amines. Examples of synthesis of poly $(N$-methyl vinylamine) (PMVAm) from poly( $N$-methyl vinylacetamide) (PNMVA) are scarce ${ }^{34,35}$ due to the difficulties of hydrolyzing secondary amides. In reasonable timescales ( $<3$ days), only $44 \%$ conversion of amides was achieved using $\mathrm{HCl} 4 \mathrm{~N}$ at $100^{\circ} \mathrm{C}$ leading to random amines/amides containing copolymers. ${ }^{34}$

This work reports a simple synthetic approach to move on from simple primary poly(vinylamine)s towards novel copolymers bearing large and predictable amounts of pendant primary and secondary amines as well as imidazole. The two-step strategy, depicted in Scheme 2, consists in the binary or ternary free radical copolymerization of NVA, NMVA and/or 1-vinylimidazole (VIm), followed by acidic hydrolysis. Special attention was paid to the determination of the comonomers reactivity ratios ${ }^{36}$ by the FinemanRoss (FR), ${ }^{37}$ Kelen-Tudos (KT) ${ }^{38}$ and the non-linear least square fitting curve (NL) ${ }^{39,40}$ methods. The Skeist's model ${ }^{41}$ was also used for controlling the chemical composition of the copolymers which changes with the monomer conversion. Based on the reactivity ratios, we also prepared a series of P(NVA-stat-NMVA-stat-VIm) terpolymers whose composition could be precisely predicted according to the Alfrey and Goldfinger's equation ${ }^{42}$. Finally, an efficient hydrolysis procedure was applied in order to convert efficiently the $N$-methyl vinylacetamide into secondary amines and produce the targeted poly(vinyl amine) copolymers.

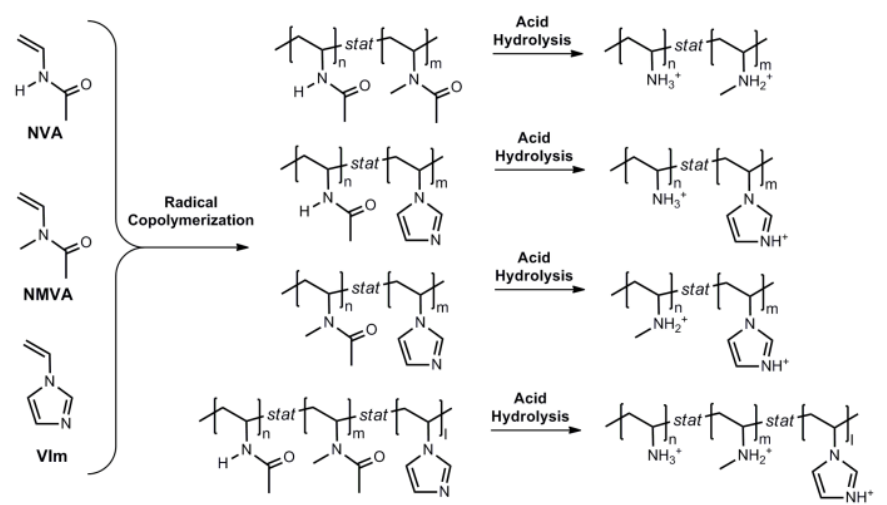

Scheme 2. General strategy for the synthesis of poly(vinylamine)copolymers .

\section{Experimental Section}

Materials. $N$-vinylacetamide (NVA) (> 98\%, TCI) was used as received. $N$-methylvinylacetamide (NMVA) (> 98\% Aldrich) and 1vinylimidazole (VIm) (> $99 \%$, Aldrich) were purified by distillation under reduced pressure. Methanol was degassed by bubbling argon for 30min. 2,2'-Azobis(4-methoxy-2,4-dimethyl valeronitrile) (V-70, $\mathrm{t}_{1 / 2}=10 \mathrm{~h}$ at $\left.30^{\circ} \mathrm{C}\right)(>98 \%$, Wako) was used as received. Dialysis membranes (cut off $1 \mathrm{kDa}$ ) were purchased from VWR.
Characterization. The (co)polymers were characterized by size exclusion chromatography (SEC) at $55^{\circ} \mathrm{C}$ in dimethylformamide (DMF) containing $\mathrm{LiBr}(0.025 \mathrm{M})$ using a flow rate at $1 \mathrm{~mL} / \mathrm{min}$. SEC curves were recorded with a Waters chromatograph equipped with three columns (Waters Styragel pss gram $1000 \AA$ (×2), 30 $)$, a dual $\lambda$ absorbance detector (Waters 2487) and a refractive index detector (Waters 2414). Multi-angle laser light scattering (MALLS) measurements were performed with a Dawn Heleos apparatus from Wyatt Technology to determine the absolute average number molar masses $\left(M_{n}\right)$ and average weight molar masses $\left(M_{w}\right)$. Data were processed with Astra V Software (Wyatt technology). In this case, $\mathrm{dn} / \mathrm{dc}$ values were measured by refractometry analysis using a Wyatt Optilab rEX refractive index detector $(\lambda=658 \mathrm{~nm})$. Mn and molar mass dispersities (Đ) were also determined using a polystyrene calibration.

The poly(vinylamine) (PNVAm) and $\operatorname{poly}(N$-methyl vinylamine) (PNMVAm) homopolymers were analyzed by SEC in water containing $\mathrm{NaCl}(0.1 \mathrm{M})$ and TFA $(0.1 \% \mathrm{v})$ at $30^{\circ} \mathrm{C}$ (flow rate: $1 \mathrm{ml} / \mathrm{min}$ ) using a Waters SEC equipped with a pre-column (PSS NOVEMA Max analytical 10 micron, $8.0 \times 50 \mathrm{~mm}$ ) and a linear column (PSS NOVEMA Max analytical linear S micron $8.0 \times 300$ $\mathrm{mm})$. Poly(2-vinylpyridine) (P2VP) standards were used to generate the calibration plot.

Infrared-spectra were recorded with a Thermo Scientific Nicolet IS5 apparatus.

${ }^{1} \mathrm{H}$ NMR spectra were recorded at $298 \mathrm{~K}$ with Burker spectrometer $(400 \mathrm{MHz})$ and treated with MestraNova software. Heteronuclear Single Quantum Correlation (HSQC) spectra were recorded at $298 \mathrm{~K}$ on a Bruker Avance $(500 \mathrm{MHz})$ instrument in $\mathrm{D}_{2} \mathrm{O}$ chemical shifts are reported in $\delta$ values (ppm) relative to internal TMS. 2D Spectra were treated with TopSpin. Elementary analyses were carried out by Service de Microanalyse ICSN. - CNRS (Gif sur Yvette, France) to determine the $\mathrm{C} / \mathrm{N}$ ratios in the copolymers before and after acidic treatment in order to calculate the hydrolysis level of the amide functions.

General copolymerization procedure for reactivity ratios determination. For each pair of comonomers (NVA-NMVA, NVAVIm and NMVA-VIm), at least seven copolymerization experiments were carried out under the same conditions with various feed compositions (molar fraction $(f)$ ranging from 0.05 and 0.95 ) (See Table S1). Typically, copolymerizations were performed under argon in a Schlenck tube by dissolving V70 (0.02 equivalents compared to the comonomers) and various amounts of comonomers (NVA, NMVA and VIm) in MeOD (8.8 mmol of comonomers/ $\mathrm{mL}$ of MeOD) containing traces of dimethylsulfoxide $\left(\mathrm{V}_{\mathrm{DMSO}} / \mathrm{V}_{\mathrm{MeOD}}=\right.$ 0.05). MeOD was used as solvent in order to easily evaluate the initial and instantaneous composition of the mixture by ${ }^{1} \mathrm{H}$ NMR whereas small quantities of DMSO served as internal calibration for monitoring the monomer conversion along the polymerization. The reaction medium was heated at $30^{\circ} \mathrm{C}$ and the polymerization was stopped below $20 \%$ of monomer conversion. The copolymers were purified by precipitation (in acetone for P(NVA-stat-VIm) and $\mathrm{P}(\mathrm{NVA}$-stat-NMVA) and in diethylether for P(NMVA-stat-VIm), collected by filtration and dried under vacuum at $40{ }^{\circ} \mathrm{C}$. The molar fraction of each comonomer in the copolymer $(F)$ was determined by ${ }^{1} \mathrm{H}$ NMR in MeOD. Polymerization time, conversion, initial feed composition and copolymer composition are presented in Table S1. These data were used to determine the reactivity ratios according to the Fineman-Ross (FR), ${ }^{37}$ Kelen-Tudos (KT) ${ }^{38}$, Extended KelenTudos $(\mathrm{EKT})^{43}$ and the non-linear (NL) ${ }^{39,40}$ least square fitting curve methods (see Table 1). Compositional drifts were also evaluated according to the Skeist's equation. ${ }^{41}$ 
Synthesis of P(NVA $\mathbf{A}_{0.68}$-stat $\left.-\mathrm{NMVA}_{\mathbf{0 . 3 2}}\right)$. NVA $(5.75 \mathrm{~g}, 67.5 \mathrm{mmol})$ and V70 (416 mg, $1.3 \mathrm{mmol})$ were placed in a flask under argon and dissolved in degassed methanol $(15 \mathrm{~mL})$ before addition of distilled and degassed NMVA $(7 \mathrm{~mL}, 6.7 \mathrm{~g}, 67.7 \mathrm{mmol})$. The reaction mixture $\left(f_{\mathrm{NVA}}=0.50, f_{\mathrm{NMVA}}=0.50\right)$ was heated at $30^{\circ} \mathrm{C}$ for 30 minutes and the polymerization was stopped at low global molar monomer conversion (32\%). The copolymer was first purified by precipitation in acetone, filtrated and dried under vacuum. Then, the copolymer was solubilized in water and purified by dialysis (membrane cut off $1 \mathrm{kDa}$ ) against water for two days before lyophilization. P(NVA-stat-NMVA) was collected as a white powder and characterized by ${ }^{1} \mathrm{H}$ NMR in $\mathrm{D}_{2} \mathrm{O}$ and SEC-MALLS in DMF $\left(\mathrm{dn} / \mathrm{dc}=0.084, \mathrm{M}_{\mathrm{n} \text { MALLS }}=35100 \mathrm{~g} / \mathrm{mol}, \mathrm{M}_{\mathrm{n} \text { cal PS }}=38160\right.$ $\left.\mathrm{g} / \mathrm{mol}, \mathrm{Ð}_{\text {cal PS }}=1.73, F_{\mathrm{NVA}}=0.68, F_{\mathrm{NMVA}}=0.32\right)$. (See Table 3$)$

Synthesis of $\mathbf{P}\left(\mathbf{N V A}_{\mathbf{0 . 5 2}}\right.$-stat-VIm (.48 $)$. NVA (2.67 g, $\left.31.4 \mathrm{mmol}\right)$ and V70 (165 mg, $0.5 \mathrm{mmol})$ were placed under argon in a flask, dissolved in degassed methanol $(6 \mathrm{~mL})$ and added with distilled and degassed 1-VIm (1.9 mL, $1.98 \mathrm{~g}, 21.0 \mathrm{mmol})$. The polymerization medium $\left(f_{\mathrm{NVA}}=0.59, f_{\mathrm{VIm}}=0.41\right)$ was heated at $30^{\circ} \mathrm{C}$ for 30 minutes (total molar monomer conversion measured by ${ }^{1} \mathrm{H} N M R$ in $\mathrm{D}_{2} \mathrm{O}=21$ $\%)$. The copolymer was first purified by precipitation in acetone, filtrated and dried under vacuum. Then, the copolymer was solubilized in water and purified by dialysis (membrane cut off $1 \mathrm{kDa}$ ) against water for three days before lyophilization. P(NVAstat-VIm) was collected as a white powder and characterized by ${ }^{1} \mathrm{H}$ NMR in $\mathrm{D}_{2} \mathrm{O}\left(F_{\mathrm{NVA}}=0.52, F_{\mathrm{VIm}}=0.48\right)$. (See Table 3$)$

Synthesis of P(NMVA $\mathbf{0 . 5 4}_{\mathbf{0}}$-stat-VIm $\left.{ }_{\mathbf{0 . 4 6}}\right)$. V70 (416 mg, $1.3 \mathrm{mmol}$ ) was placed under argon in a flask, dissolved in degassed methanol $(15 \mathrm{~mL})$ followed by addition with a syringe of distilled and degassed NMVA (10.5 mL, $10.07 \mathrm{~g}, 101.6 \mathrm{mmol})$ and 1 -VIm ( $3 \mathrm{~mL}$, $3.12 \mathrm{~g}, 33.7 \mathrm{mmol})$. The reaction mixture $\left(f_{\mathrm{NMVA}}=0.73, f_{\mathrm{VIm}}=0.27\right)$ was then heated at $30^{\circ} \mathrm{C}$ for $2 \mathrm{~h}$ (total monomer conversion measured by ${ }^{1} \mathrm{H}$ NMR in $\mathrm{D}_{2} \mathrm{O}=29 \%$ ). The copolymer was first purified by precipitation in diethyl ether, filtrated and dried under vacuum. Next, the copolymer was solubilized in water and purified by dialysis (membrane cut off $1 \mathrm{kDa}$ ) against water for three days before lyophilization. P(NMVA-stat-VIm) was collected as a white solid and characterized by ${ }^{1} \mathrm{H}$ NMR in $\mathrm{D}_{2} \mathrm{O}$ and SEC-MALLS in DMF $\left(\mathrm{d}_{\mathrm{n}} / \mathrm{d}_{\mathrm{c}}=0.073, \mathrm{M}_{\mathrm{n} \text { MALLS }}=18000 \mathrm{~g} / \mathrm{mol}, \mathrm{M}_{\mathrm{n} \text { cal PS }}=20060 \mathrm{~g} / \mathrm{mol}, \bigoplus_{\text {cal }}\right.$ $\left.\mathrm{PS}=2.37, F_{\mathrm{NMVA}}=0.54, F_{\mathrm{VIm}}=0.46\right) .($ See Table 3$)$

Synthesis of $\mathbf{P}\left(\mathbf{N V A}_{\mathbf{0 . 3 2}}\right.$-stat-NMVA s.34-stat-VIm $\left._{\mathbf{0 . 3 4}}\right)$. NVA (2.47 g, $29 \mathrm{mmol})$ and $\mathrm{V} 70(416 \mathrm{mg}, 1.3 \mathrm{mmol})$ were placed under argon and dissolved by a degassed methanol $(15 \mathrm{~mL})$ followed by addition with a syringe of distilled and degassed 1-Vim $(1.8 \mathrm{~mL}, 1.84 \mathrm{~g}, 19.6$ mmol) and NMVA $(8.9 \mathrm{~mL}, 8.53 \mathrm{~g}, 86.4 \mathrm{mmol})$. The reaction medium $\left(f_{\mathrm{NVA}}=0.19, f_{\mathrm{NMVA}}=0.64, f_{\mathrm{VIm}}=0.17\right)$ were added and the mixture was heated at $30^{\circ} \mathrm{C}$ for $2 \mathrm{~h}$ (total monomer conversion measured by ${ }^{1} \mathrm{H}$ NMR in $\mathrm{D}_{2} \mathrm{O}=28 \%$ ). The copolymer was first purified by precipitation in acetone, filtrated and dried under vacuum. Then, the copolymer was solubilized in water and dialyzed (membrane cut off $1 \mathrm{kDa}$ ) against pure water for three days before lyophilization. P(NVA-stat-NMVA-stat-VIm) was collected as an off-white solid and characterized by ${ }^{1} \mathrm{H}$ NMR in $\mathrm{D}_{2} \mathrm{O}$ and SECMALLS in DMF $\left(\mathrm{d}_{\mathrm{n}} / \mathrm{d}_{\mathrm{c}}=0.083, \mathrm{M}_{\mathrm{n} \text { MALLS }}=38700 \mathrm{~g} / \mathrm{mol}, \mathrm{M}_{\mathrm{n} \text { cal PS }}=\right.$ $\left.25470 \mathrm{~g} / \mathrm{mol}, \bigoplus_{\text {cal PS }}=1.75, F_{\mathrm{NVA}}=0.32, F_{\mathrm{NMVA}}=0.34, F_{\mathrm{VIm}}=0.34\right)$. (See Table 3) In general, the compositions of the terpolymers were successfully predicted based on the Alfrey-Goldfinger (AG) ${ }^{42}$ equation (See Table 2).

Synthesis of PNVA. NVA (4 g, $47 \mathrm{mmol})$ and V70 (320 mg, 1 $\mathrm{mmol}$ ) were placed under argon and dissolved in degassed methanol $\left(6 \mathrm{~mL}\right.$ ). The reaction was heated at $30^{\circ} \mathrm{C}$ for 45 minutes (total monomer conversion measured by ${ }^{1} \mathrm{H} N \mathrm{NM}$ in $\mathrm{D}_{2} \mathrm{O}=35 \%$ ). The polymer was purified by precipitation in acetone, filtrated and dried under vacuum before dissolution in water and dialysis (membrane cut off $1 \mathrm{kDa}$ ) against pure water for two days. After lyophilization, PNVA was collected as a white solid and characterized by ${ }^{1} \mathrm{H}$ NMR in $\mathrm{D}_{2} \mathrm{O}$ and SEC-MALLS in DMF $\left(\mathrm{d}_{\mathrm{n}} / \mathrm{d}_{\mathrm{c}}=0.060, \mathrm{M}_{\mathrm{n} \text { MALLS }}=58300\right.$ $\left.\mathrm{g} / \mathrm{mol}, \mathrm{M}_{\mathrm{n} \text { cal PS }}=31300 \mathrm{~g} / \mathrm{mol}, \bigoplus_{\text {cal PS }}=1.69\right)$. (See Table 3)

Synthesis of PNMVA. V70 (416 mg, $1.35 \mathrm{mmol}$ ) was placed under argon in a flask, dissolved in degassed methanol $(14 \mathrm{~mL})$ followed by addition with a syringe of distilled and degassed NMVA $(14 \mathrm{~mL}$, $13.4 \mathrm{~g}, 135 \mathrm{mmol})$. The reaction mixture was then heated at $30^{\circ} \mathrm{C}$ for 4h (total monomer conversion measured by ${ }^{1} \mathrm{H}$ NMR in $\mathrm{D}_{2} \mathrm{O}=14$ $\%)$. The polymer was then purified by precipitation in diethyl ether, filtrated and dried under vacuum before dissolution in water and dialysis (membrane cut off $1 \mathrm{kDa}$ ) against water for two days. After lyophilization, PNMVA was collected as a white solid and characterized by ${ }^{1} \mathrm{H}$ NMR in $\mathrm{D}_{2} \mathrm{O}$ and SEC-MALLS in DMF $\left(\mathrm{d}_{\mathrm{n}} / \mathrm{d}_{\mathrm{c}}=\right.$ $0.070, \mathrm{M}_{\mathrm{n} \text { MALLS }}=13650 \mathrm{~g} / \mathrm{mol}, \mathrm{Mn}_{\text {cal PS }}=17780 \mathrm{~g} / \mathrm{mol}, \bigoplus_{\text {cal PS }}=$ 1.79). (See Table 3 )

General procedure for hydrolysis of the amide-containing (co)polymers. The copolymer $(400 \mathrm{mg})$ was dissolved in $\mathrm{HCl} 6 \mathrm{~N}$ $(5.5 \mathrm{~mL})$. The resulting solution of (co)polymer $(7 \% \mathrm{w} / \mathrm{v})$ was then placed in an Acid Digestion Parr Vessel and heated at $120^{\circ} \mathrm{C}$ for 64h. After cooling, the solution was dialyzed against pure distilled water for one night. During the last two hours of dialysis, the $\mathrm{pH}$ was maintained at $7 \mathrm{pH}$ controlled with $\mathrm{pH}$ meter and adjusted by addition of $\mathrm{NaOH} 0.1 \mathrm{~N}$ or $\mathrm{HCl} 1 \mathrm{~N}$ ). The (co)polymers was recovered upon lyophilization. The (co)polymer was analyzed by ${ }^{1} \mathrm{H}$ NMR in $\mathrm{D}_{2} \mathrm{O}$, IR and EA. Hydrolysis yields were determined by EA comparing the $\mathrm{C} / \mathrm{N}$ content ratio in the (co)polymer before and after acidic treatment. All results are presented in Table 3. Aqueous SEC experiments were also performed on the PNVAm and PNMVAm samples. In addition, the hydrolysis of PNVA and PNMVA was also tested under milder conditions $\left(5 \% \mathrm{w} / \mathrm{v}, 2 \mathrm{~N} \mathrm{HCl}, 120^{\circ} \mathrm{C}, 14 \mathrm{~h}\right)$.

\section{Results and Discussion}

\section{Binary copolymerization}

The synthesis of copolymers bearing various pendant amines starts with the free radical copolymerization of the vinylamides, NVA, NMVA and VIm (see Scheme 2). In order to predict the monomer composition in the final copolymers and the comonomer sequence along the backbone, we investigated the free radical copolymerization of these comonomers in pairwise combinations and measured their reactivity ratios. For this purpose, at least seven copolymerization experiments were conducted for each binary system with different feed compositions ranging from 0.10 to 0.95 (Table S1). Typically, copolymerizations were performed at $30^{\circ} \mathrm{C}$ in $\mathrm{MeOD}$ using V70 as radical initiator. A deuterated solvent was used in order to determine precisely the initial comonomer feed by ${ }^{1} \mathrm{H}$ NMR and to monitor the conversion by following the loss of intensity of the olefinic protons of each monomer. Low monomer conversions $(<20 \%)$ were targeted to prevent significant composition drift. After purification of the copolymers, their compositions were analyzed by ${ }^{1} \mathrm{H}$ NMR. Representative spectra of $\mathrm{P}(\mathrm{NVA}$-stat-NMVA), P(NVA-stat-VIm) and P(NMVA-stat-VIm) are provided in Figure S1. The molar fractions of the comonomers in the copolymer $(F)$ were then plotted as a function of their molar fraction in the feed $(f)$ and fitted with the Mayo-Lewis equation (Figure 1). 


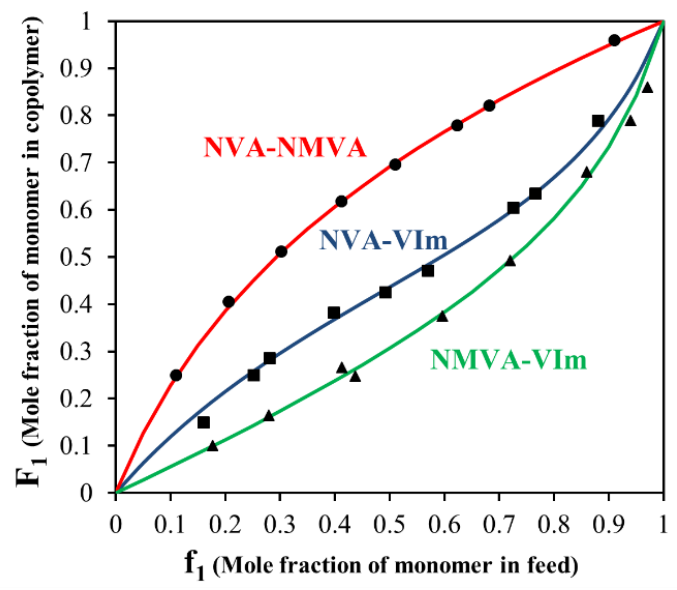

Fig. 1. Relationship between the molar fraction of monomer in the feed and in the copolymer for poly $(N$-vinylacetamide-stat- $N$-methyl vinylacetamide) $(\bullet), \operatorname{poly}(N$-vinylacetamide-stat-1-vinylimidazole $)$ (a) $\operatorname{poly}(N$-methyl vinylacetamide -stat-1-vinylimidazole) $(\boldsymbol{\Delta})$. The full lines represent the fitting curves obtained by the least square method.

Based on these data, the reactivity ratios of each monomer pair were measured by different methods. First, the classical Fineman-Ross calculation was used (eq.1). ${ }^{37}$ For all copolymerizations, we obtained straight lines whose slope and intercept with the ordinate ( $\mathrm{Y}$-axes) corresponds to $r_{1}$ and $-r_{2}$, respectively (Figure 2A). Reactivity ratio values are presented in Table 1 .

$$
\begin{aligned}
& f(F-1) / F=r_{1}\left(f^{2} / F\right)-r_{2} \\
& \text {, where } f=f_{1} / f_{2} \text { and } F=F_{1} / F_{2}
\end{aligned}
$$

In addition, a second linearization method, proposed by Kelen and Tudos, ${ }^{38}$ was tested. The latter involves parameters $\eta$ and $\zeta$, mathematical functions of the mole ratios in the monomer feed $(f)$ and in the copolymer $(F)$ and of a parameter $\alpha$ calculated on the basis of the lowest and highest values of $\left(\mathrm{f}^{2} / \mathrm{F}\right)$. Figure $2 \mathrm{~B}$ shows linear plots of $\eta$ as a function of $\zeta$ leading to $r_{1}$ and $\left(-r_{2} / \alpha\right)$ via the intercepts at $\xi=1$ and $\xi=0$, respectively.

$$
\begin{aligned}
\eta=\left(\mathrm{r}_{1}+\left(\mathrm{r}_{2} / \alpha\right)\right) \zeta-\left(\mathrm{r}_{2} / \alpha\right) \\
\quad, \text { where } \eta=(\mathrm{f}(\mathrm{F}-1)) /\left(\mathrm{F}\left(\alpha+\left(\mathrm{f}^{2} / \mathrm{F}\right)\right) ; \zeta=\left(\mathrm{f}^{2} / \mathrm{F}\right) /\left(\alpha+\left(\mathrm{f}^{2} / \mathrm{F}\right)\right)\right. \\
\quad ; \alpha=\left(\left(\mathrm{f}^{2} / \mathrm{F}\right)_{\max } \times\left(\mathrm{f}^{2} / \mathrm{F}\right)_{\min }\right)^{0.5}
\end{aligned}
$$

Finally, reactivity ratios were determined by a non-linear least squares fitting curve $\mathrm{c}^{39,40}$ method according to the Mayo-Lewis equation (eq. 3) in order to avoid inaccuracy inherent to the linearization process. The fitting curves are presented for each comonomer pair in Figure 1 and the resulting $r_{1}$ and $r_{2}$ values calculated accordingly are presented with those obtained by the FR and KT methods in Table 1.

$F_{1}=\left(r_{1} f_{1}^{2}+f_{1} f_{2}\right) /\left(r_{1} f_{1}^{2}+2 f_{1} f_{2}+r_{2} f_{2}^{2}\right)$

Although monomer conversions are low, the Extended Kelen-Tudos $(E K T)^{43}$ was also considered in order to take into account the possible composition drift along the polymerization (Table S2 and Figure S2) but very similar reactivity ratios were obtained as compared to the three other methods (compare Table 1 and Table S2).

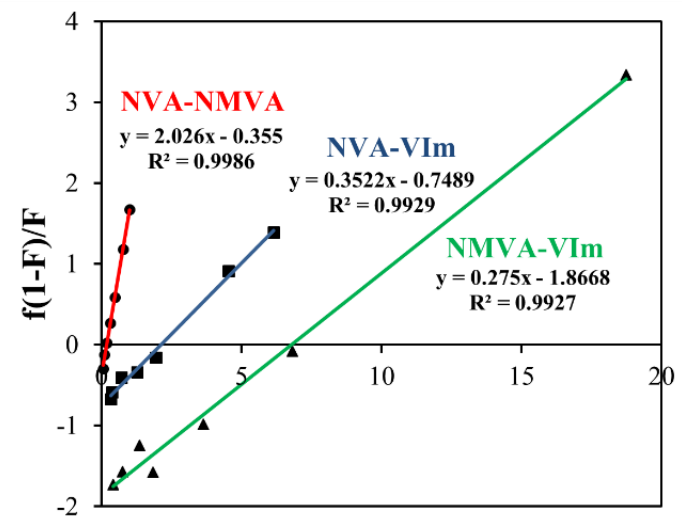

A)

$\mathbf{f}^{2} / \mathbf{F}$

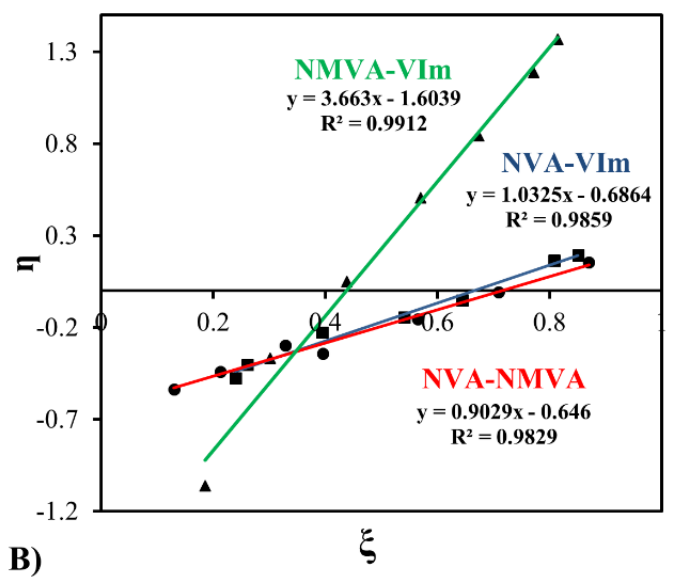

Fig. 2. Fineman-Ross (A) and Kelen-Tudos plots for the copolymerization at $30{ }^{\circ} \mathrm{C}$ of $\mathrm{N}$-vinylacetamide and $\mathrm{N}$ methylvinylacetamide) $(\bullet), N$-vinylacetamide and 1 -vinylimidazole $(\boldsymbol{\square}), N$-methylvinylacetamide and 1-vinylimidazole) ( $\mathbf{\Delta})$.

The reactivity ratios clearly suggest $\left(\mathrm{e}_{\mathrm{gha}} \mathrm{q}^{\mathrm{a}}\right)$ the monomers are preferentially consumed according to the following trend: VIm > NVA > NMVA. In the case of the $N$-vinylamides copolymerization, the insertion of NVA within the chain is favored compared to NMVA. The slightly higher steric hindrance of the latter might contribute to this trend but little electronic effects cannot be excluded. The incorporation of NMVA is also disfavored in its copolymerization with VIm, for which the content of VIm in the resulting copolymer is always superior to the one of the feed. At last, the NVA-VIm copolymerization is slightly in favor of VIm and exhibits the highest tendency to alternation as indicated by the rather low $\mathrm{r}_{1} \times \mathrm{r}_{2}$ value $(\sim 0.25)$ and reactivity ratios below one. Hydrogen bonding between the $\mathrm{N}-\mathrm{H}$ proton of the acestamide group and the imidazole ring might account for this alternation propensity.

Table 1. Calculated monomer reactivity ratios.

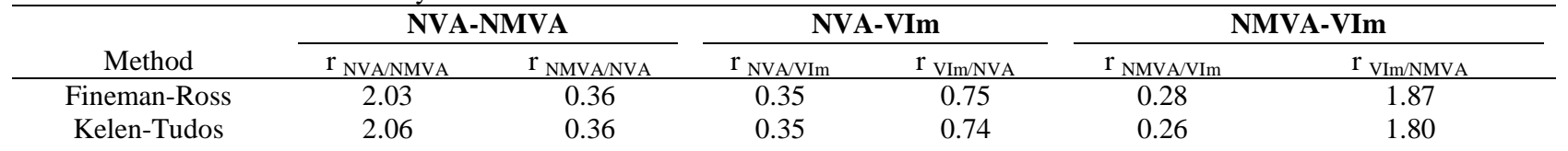




\begin{tabular}{ccccccc} 
Nonlinear & 1.98 & 0.33 & 0.35 & 0.74 & 0.27 & 1.85 \\
\hline Average & 2.02 & 0.35 & 0.35 & 0.74 & 0.27 & 1.84 \\
\hline
\end{tabular}

Considering the important differences in reactivity ratios, the comonomers are consumed at different rates in the copolymerizations, and consequently the monomer feed composition changes significantly in the course of the copolymerization. As chains are created and terminate continuously in free radical polymerization, this results in an inhomogeneity in composition of the chains distribution. In order to get a good compositional picture of the copolymer distribution, the instantaneous and the cumulative copolymer compositions were predicted on the whole range of the monomer conversion with the Skeist's model (SK) (eq. 4) using the average reactivity ratios determined beforehand. The cumulative composition is given by equation 5 .

$$
\text { Conv }=1-\left(\mathrm{M} / \mathrm{M}_{0}\right)=\left(\mathrm{f}_{1} / \mathrm{f}_{1}{ }^{\circ}\right)^{\alpha}\left(\mathrm{f}_{2} / \mathrm{f}_{2}{ }^{\circ}\right)^{\beta}\left[\left(\mathrm{f}_{1}{ }^{\circ}-\delta\right) /\left(\mathrm{f}_{1}{ }^{\circ}-\delta\right)\right]^{\gamma}
$$

, where $\mathrm{M}_{0}$ and $\mathrm{M}$ are the initial and the instantaneous monomer concentration, $f^{\circ}$ and $f$ correspond to the initial and the instantaneous mole fraction in the feed and $\alpha, \beta, \delta, \gamma$ are defined as follows: $\alpha=\mathrm{r}_{2} /\left(1-\mathrm{r}_{2}\right) ; \beta=\mathrm{r}_{1} /\left(1-\mathrm{r}_{1}\right) ; \gamma=(1-$ $\left.r_{1} r_{2}\right) /\left(1-r_{1}\right)\left(1-r_{2}\right) ; \delta=\left(1-r_{2}\right) /\left(2-r_{1}-r_{2}\right)$

$\mathrm{F}_{1 \text { cumul }}=\left(\mathrm{f}_{1}{ }^{\circ}-\mathrm{f}_{1}(1-\mathrm{conv}).\right) /$ conv.

As an illustration, Figure 3 shows the evolution of the instantaneous feed and copolymer composition as well as the cumulative copolymer composition as a function of the overall conversion when starting from a near-stoichiometric comonomer feed composition. All copolymerizations were performed at $30{ }^{\circ} \mathrm{C}$ in methanol and samples were withdrawn regularly from the medium along the polymerization. The cumulative copolymer compositions measured by ${ }^{1} \mathrm{H}$ NMR at different conversions fitted well with the predicted cumulative composition curve (eq. 5), which confirms the validity of the model and the determined reactivity ratios. For the NVA-NMVA and NMVA-VIm copolymerizations, the instantaneous composition changes only slightly up to $25 \%$ of monomer conversion, but then evolves drastically. In contrast, the instantaneous composition of the P(NVA-stat-VIm) copolymers changes little up to almost $75 \%$ of conversion. This model thus provides important information on the polymer sequence, particularly on the homogeneity of the composition over the chain distribution that is worth to be taken into account when considering the synthesis of this type of copolymers. Interestingly, in case of a controlled radical copolymerization (CRP), a composition drift of the feed during the polymerization leads to gradient copolymers showing an evolution of their composition along the backbone. Since reactivity ratios are often very similar for conventional and controlled radical copolymerizations performed under the same experimental conditions, the compositional drifts determined above by the Skeist's method are also useful to predict the gradient composition of the same copolymers prepared by controlled radical polymerization techniques. Of course, such calculations could be performed for any initial comonomer feed ratio in order to anticipate and prevent significant inhomogeneity in composition of the chains. It is important to note that, controlling the (co)polymerization of $\mathrm{N}$-vinylamides and VIm remains hitherto quite a challenge. As an example, the organometallic-mediated radical polymerization (OMRP) was proved to be very efficient for controlling the polymerization of several N-vinylamides, ${ }^{44-47}$ except NVA, whereas the OMRP of VIm has not been reported yet. To the best of our knowledge, no CRP method can manage the simultaneous control of these three monomers yet.

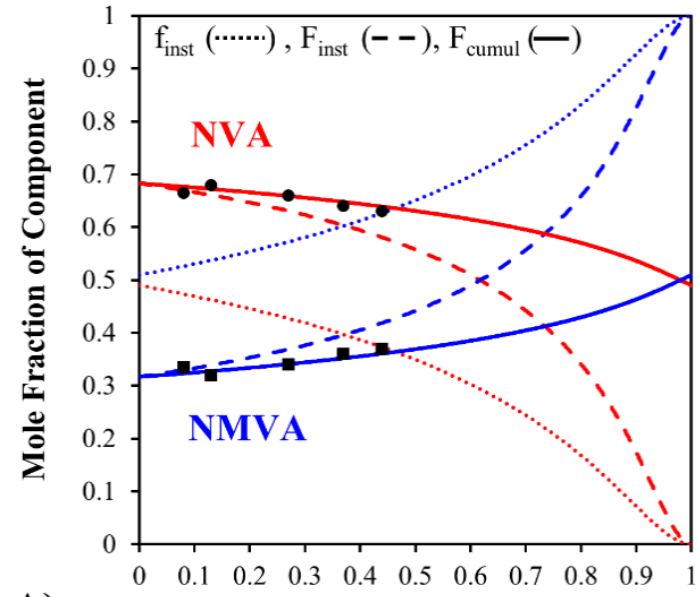

A)

Overall Monomer Conversion
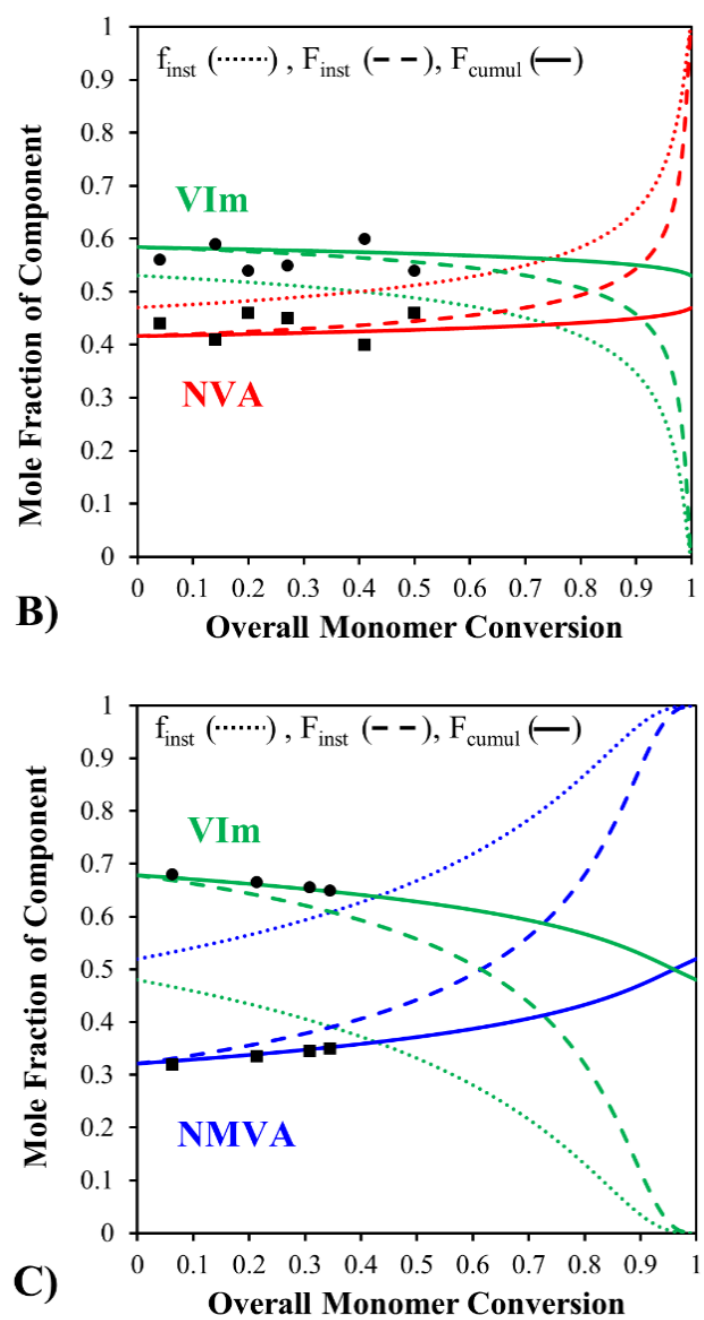

Figure 3. Instantaneous mole fraction of monomer in the feed $\left(f_{\text {inst }}\right.$ ...) as well as instantaneous $\left(\mathrm{F}_{\text {inst }}---\right)$ and cumulative $\left(\mathrm{F}_{\text {cumul }}-\right)$ mole fractions in the copolymer vs. the overall molar monomer conversion calculated based on Skeist's model when starting from near equimolar mixtures of comonomers. Experimental data of the 
average copolymer composition $(\bullet, \boldsymbol{\bullet})$ were determined by ${ }^{1} \mathrm{H}$ NMR.

\section{Ternary copolymerization}

After studying the binary copolymerization systems, we investigated the terpolymerization of NVA, NMVA and VIm under similar experimental conditions. Copolymerizations were initiated with V70 in methanol at $30{ }^{\circ} \mathrm{C}$ and the monomer conversions were kept below $20 \%$. Table 2 compares the actual and theoretical copolymer compositions for different comonomer feed compositions. The predicted compositions were calculated based on the AlfreyGoldfinger $(\mathrm{AG})^{42}$ equation (eq. 6), using the average reactivity ratios summarized in Table 1.

$\mathrm{d}\left[\mathrm{M}_{1}\right]: \mathrm{d}\left[\mathrm{M}_{2}\right]: \mathrm{d}\left[\mathrm{M}_{3}\right]=$

$\left[M_{1}\right]\left\{\left[M_{1}\right] / r_{31} \cdot r_{21}+\left[M_{2}\right] / r_{21} \cdot r_{32}+\left[M_{3}\right] / r_{31} \cdot r_{23}\right\}\left\{\left[M_{1}\right]+\left[M_{2}\right] / r_{12}+\left[M_{3}\right] / r_{13}\right\}:$

$\left[\mathrm{M}_{2}\right]\left\{\left[\mathrm{M}_{1}\right] / \mathrm{r}_{12} \cdot \mathrm{r}_{31}+\left[\mathrm{M}_{2}\right] / \mathrm{r}_{12} \cdot \mathrm{r}_{32}+\left[\mathrm{M}_{3}\right] / \mathrm{r}_{32} \cdot \mathrm{r}_{13}\right\}\left\{\left[\mathrm{M}_{2}\right]+\left[\mathrm{M}_{1}\right] / \mathrm{r}_{21}+\left[\mathrm{M}_{3}\right] / \mathrm{r}_{23}\right\}:$

$\left[\mathrm{M}_{3}\right]\left\{\left[\mathrm{M}_{1}\right] / \mathrm{r}_{13} \cdot \mathrm{r}_{21}+\left[\mathrm{M}_{2}\right] / \mathrm{r}_{23} \cdot \mathrm{r}_{12}+\left[\mathrm{M}_{3}\right] / \mathrm{r}_{13} \cdot \mathrm{r}_{23}\right\}\left\{\left[\mathrm{M}_{3}\right]+\left[\mathrm{M}_{1}\right] / \mathrm{r}_{31}+\left[\mathrm{M}_{2}\right] / \mathrm{r}_{32}\right\}$

Generally, the compositions of the terpolymers measured by ${ }^{1} \mathrm{H}$ NMR were close to the prediction. The slight discrepancies between the values might be due to complex penultimate effects that are not taken into account in the used theories. Nevertheless, the prediction tool is valid and of practical importance for this ternary copolymerization. Moreover, the data collected in Table 2 confirms the trends observed in the binary systems: VIm and NMVA are the most and the least reactive monomers, respectively. Overall, the compositional prediction and thus control of the P(NVA-statNMVA-stat-VIm) terpolymers is now possible. Indeed, comonomer reactivity ratios combined with the AG model allow determining the amount of each comonomers to be introduced in the initial monomer feed in order to target a tercopolymer with a specific composition. Overall, this study makes the synthesis of such copolymers highly efficient and paves the way for further innovation based thereon.

\section{Hydrolysis of poly(vinylamide) derivatives.}

The hydrolysis of poly( $N$-vinylamide $) s$ has been previously investigated in the literature as a route to the corresponding pendant amine polymers used in many industrial applications. ${ }^{8-10,12,13}$ Among the PVAm precursors, PNVF is certainly the most popular because of the ease of hydrolysis of the formamide moieties. ${ }^{17-19}$ Nevertheless, as reported by Akashi et al, ${ }^{20,21}$ near complete removal of the acetamide groups of PNVA can also be achieved by hydrochloric treatment $(\mathrm{HCl} 2 \mathrm{~N})$ at $120{ }^{\circ} \mathrm{C}$ for $12 \mathrm{~h}$. In contrast, the conversion of PNMVA into the corresponding pendant secondary amine polymer is more difficult and requires extremely long reaction times (around $150 \mathrm{~h}) .{ }^{35}$ For shorter timescales $(60 \mathrm{~h})$ only moderate hydrolysis of PNMVA (44\%) was achieved upon treatment of PNMVA with $\mathrm{HCl} 4 \mathrm{~N}$ at $100^{\circ} \mathrm{C} .^{34}$

In order to produce polymers with high amine contents, we first optimized the hydrolysis conditions of PNVA and PNMVA homopolymers prior to investigating the transformation of the binary and ternary copolymers prepared in the former section. Two sets of hydrolysis conditions were tested. The first method is similar to the one reported by Akashi et $\mathrm{al}^{20,21}$, i.e. $\mathrm{HCl} 2 \mathrm{~N} / 120^{\circ} \mathrm{C} / 14 \mathrm{~h}$. Secondly, harsher conditions were considered $\left(\mathrm{HCl} 6 \mathrm{~N} / 120^{\circ} \mathrm{C} / 64\right.$ $\mathrm{h})$. The latter are inspired by the hydrolysis method reported by Okatova et $\mathrm{al}^{34}\left(4 \mathrm{~N} / 100{ }^{\circ} \mathrm{C} / 60 \mathrm{~h}\right)$ using prolonged reaction time and a higher acid concentration except for that we increased the hydrochloric acid concentration to $6 \mathrm{~N}$ in order to reach beyond the $44 \%$ of hydrolysis yield reported for the PNMVA ${ }^{34}$.

In all cases, the copolymer concentration in the $\mathrm{HCl}$ solution was rather low $(5-7 \%$ w/v). For PNVA, both procedures afforded high levels of hydrolysis, as suggested by the disappearance of signal $\mathbf{c}$ at $2.0 \mathrm{ppm}$ corresponding to the methyl protons of the acetamide group on the ${ }^{1} \mathrm{H}$ NMR spectra of the purified product (Figure 4; left spectra). In agreement with previously reported NMR characterization of PVAm, a shift of the methylene protons from 1.6 ppm (a) to 2.0 ppm (a') was observed upon hydrolysis. ${ }^{48}$ It is worth mentioning that the amine proton resonance cannot be detected because of rapid proton exchange with $\mathrm{D}_{2} \mathrm{O}$. The conversion of PNVA into PNVAm was quantified by elementary analysis (EA) by comparison of the $\mathrm{C} / \mathrm{N}$ content ratio in polymer before and after acidic treatment (Table S3). This comparative method based on the $\mathrm{C} / \mathrm{N}$ ratio rather than on absolute $\mathrm{C}$ or $\mathrm{N}$ mass percentages prevents mistakes inherent to the hygroscopic character of these polymers. PNVA hydrolysis rate as high as $90 \%$ and $94 \%$ were recorded, when using the milder $\left(\mathrm{HCl} 2 \mathrm{~N}, 120^{\circ} \mathrm{C}, 14 \mathrm{~h}\right)$ and the harsher $(\mathrm{HCl}$ $6 \mathrm{~N}, 120^{\circ} \mathrm{C}, 64 \mathrm{~h}$ ) hydrolytic conditions, respectively (Table 3 , entry 1). In other words, no significant difference in the PNVA hydrolysis is observed for the two sets of experiments.

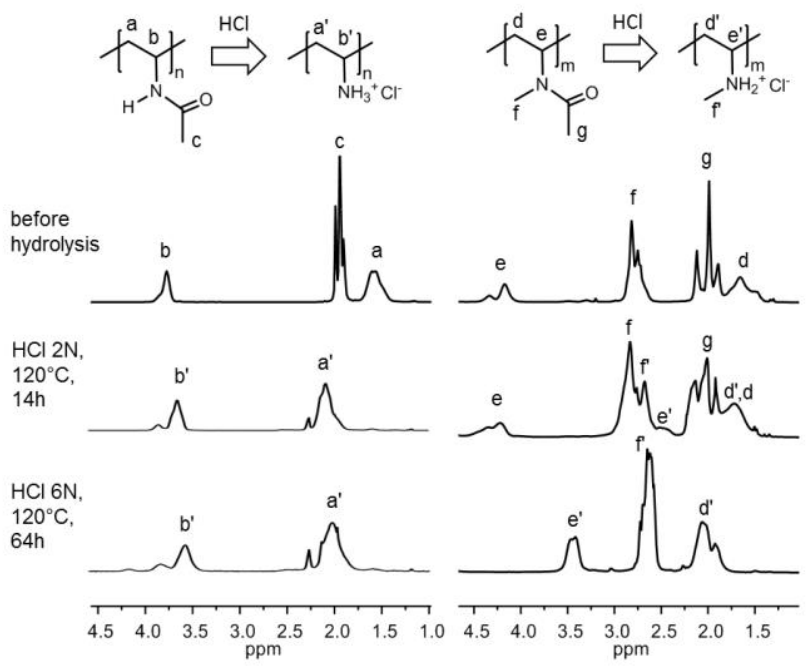

Figure 4. ${ }^{1} \mathrm{H}$ NMR of the PNVA (left) and PNMVA (right) before and after acidic hydrolysis and purification by dialysis under various conditions. Spectra were recorded at $298 \mathrm{~K}$ in $\mathrm{D}_{2} \mathrm{O}$.

Table 2. Experimental and theoretical compositions of ternary copolymers.

\begin{tabular}{|c|c|c|c|c|c|c|c|c|}
\hline & & & \multicolumn{6}{|c|}{ Terpolymer composition } \\
\hline \multicolumn{3}{|c|}{ Feed composition $^{\mathrm{a}}$} & \multicolumn{3}{|c|}{ Measured $^{b}$} & \multicolumn{3}{|c|}{ Calculated $^{\mathrm{c}}$} \\
\hline$f_{\mathrm{NVA}}$ & $f_{\text {NMVA }}$ & $f_{\mathrm{VIm}}$ & $F_{\mathrm{NVA}}$ & $F_{\text {NMVA }}$ & $F_{\mathrm{VIM}}$ & $F_{\mathrm{NVA}}$ & $F_{\text {NMVA }}$ & $F_{\mathrm{VIm}}$ \\
\hline 0.19 & 0.64 & 0.17 & 0.32 & 0.34 & 0.34 & 0.29 & 0.34 & 0.37 \\
\hline 0.33 & 0.31 & 0.35 & 0.40 & 0.07 & 0.53 & 0.37 & 0.12 & 0.51 \\
\hline 0.36 & 0.54 & 0.10 & 0.50 & 0.26 & 0.24 & 0.51 & 0.24 & 0.25 \\
\hline 0.41 & 0.58 & 0.01 & 0.65 & 0.33 & 0.02 & 0.69 & 0.27 & 0.03 \\
\hline
\end{tabular}


Polymerization at $30{ }^{\circ} \mathrm{C}$. ${ }^{\text {a }}$ Determined by ${ }^{1} \mathrm{H}$ NMR of the reaction mixture at $\mathrm{t}_{0}$ in MeOD. ${ }^{\mathrm{b}}$ Determined by ${ }^{1} \mathrm{H}$ NMR in MeOD of the isolated terpolymers. ${ }^{\mathrm{c}}$ Calculated by the Alfrey and Goldfinger's equation.

Table 3. Hydrolysis of the poly( $N$-vinylamide)-based copolymers.

\begin{tabular}{|c|c|c|c|c|c|c|c|c|c|c|}
\hline \multirow[b]{2}{*}{ Entry } & \multicolumn{3}{|c|}{ Initial copolymer composition $^{\mathrm{a}}$} & \multicolumn{2}{|c|}{${\text { Hydrolysis level }(\%)^{\mathrm{b}}}^{\mathrm{b}}$} & \multicolumn{5}{|c|}{ Hydrolyzed copolymer composition $^{c}$} \\
\hline & $F_{\mathrm{NVA}}$ & $F_{\text {NMVA }}$ & $F_{\mathrm{VIM}}$ & NVA & NMVA & $F_{\mathrm{NVA}}$ & $F_{\text {NVAm }}$ & $F_{\text {NMVA }}$ & $F_{\text {NMVAm }}$ & $F_{\mathrm{VIM}}$ \\
\hline 1 & 1 & 0 & 0 & 94 & I & 0.06 & 0.94 & 0 & 0 & 0 \\
\hline 2 & 0 & 1 & 0 & I & 81 & 0 & 0 & 0.19 & 0.81 & 0 \\
\hline 3 & 0.68 & 0.32 & 0 & $94 *$ & 90 & 0.04 & 0.64 & 0.03 & 0.29 & 0 \\
\hline 4 & 0.52 & 0 & 0.48 & 91 & I & 0.05 & 0.47 & 0 & 0 & 0.48 \\
\hline 5 & 0 & 0.54 & 0.46 & I & 83 & 0 & 0 & 0.09 & 0.45 & 0.46 \\
\hline 6 & 0.32 & 0.34 & 0.34 & $94 *$ & 75 & 0.02 & 0.30 & 0.08 & 0.26 & 0.34 \\
\hline
\end{tabular}

Hydrolysis conditions: $\mathrm{HCl} 6 \mathrm{~N} / 120{ }^{\circ} \mathrm{C} / 64 \mathrm{~h} .{ }^{a}$ Determined by ${ }^{1} \mathrm{H}$ NMR in $\mathrm{D}_{2} \mathrm{O} .{ }^{b}$ Determined by elementary analysis (see Table S3 for raw data and calculations). ${ }^{c}$ Calculated by taking into account the initial composition determined by ${ }^{1} \mathrm{H}$ NMR and the hydrolysis level determined by elementary analysis. * set to $94 \%$ according to the result for PNVA homopolymer.

In contrast, the hydrolysis of PNMVA was only effective under harsh conditions $\left(\mathrm{HCl} 6 \mathrm{~N} / 120{ }^{\circ} \mathrm{C} / 64 \mathrm{~h}\right)$. Indeed, the ${ }^{1} \mathrm{H}$ NMR spectrum after treatment of PNMVA for $14 \mathrm{~h}$ at $120^{\circ} \mathrm{C}$ with $\mathrm{HCl} 2 \mathrm{~N}$ still exhibits an intense signal $\mathbf{g}$ at $2.0 \mathrm{ppm}$ characteristic of the residual acetamide (Figure 4, right spectra). ${ }^{1} \mathrm{H}-{ }^{13} \mathrm{C}$ Heteronuclear Single-Quantum Correlation (HSQC) spectroscopy experiments (Figure S3) confirmed the low level of PNMVA hydrolysis under mild acidic conditions. Comparison of the relative intensities of the acetamide (g) and methylene (d, d') signals before and after hydrolytic treatment indicated that about $17 \%$ of the amides were hydrolyzed. This value was confirmed by elementary analysis $(19 \%$ of hydrolysis). In contrast, the severe conditions $\left(\mathrm{HCl} 6 \mathrm{~N} / 120{ }^{\circ} \mathrm{C} / 64\right.$ h) allowed almost complete conversion of PNMVA into $\operatorname{poly}(N-$ methyl vinylamine) (PNMVAm). In addition to the apparent decrease in intensity of peak $\mathbf{g}$, the signals for the methine (e) and methylene protons (d) significantly shifted on the ${ }^{1} \mathrm{H}$ NMR and HSQC spectra (Figures 4 and S3). According to EA analysis, the percentage of hydrolysis reached $81 \%$ within $64 \mathrm{~h}$ (entry 2 , Table 3 ), which makes this approach valuable for the preparation of polymers containing pendant secondary amines. Note that using similar reaction time $(\sim 60 \mathrm{~h})$ as Okatova et al ${ }^{34}$ while increasing the acid concentration from $4 \mathrm{~N}$ to $6 \mathrm{~N}$, considerably improved the hydrolysis efficiency. Finally, the integrity of the poly(vinylamine) backbone following hydrolysis was confirmed by SEC in aqueous media: monomodal size exclusion chromatograms were recorded for PNVAm and PNMVAm even after the harshest acidic treatment (Figure S4).

The optimized hydrolysis conditions $\left(6 \mathrm{~N} / 64 \mathrm{~h} / 120^{\circ} \mathrm{C}\right)$ were then applied to a series of binary and ternary copolymers containing NVA, NMVA and VIm units (entries 3-6, Table 3). For each copolymer, the efficiency of hydrolysis was quantified by EA taking into account the change in the $\mathrm{C} / \mathrm{N}$ ratio upon hydrolysis. For copolymers containing both NVA and NMVA, hydrolysis percentage of NVA was fixed to $94 \%$, the value determined for homoPNVA hydrolyzed under the same conditions (entry 1, Table $3)$. Based on this approximation, the conversion of the NMVA units in the P(NVA-stat-NMVA) (entry 3, Table 3) copolymer was $90 \%$, a value consistent with the results of $\mathrm{P}(\mathrm{NMVA})$ hydrolysis. As illustrated by the ${ }^{1} \mathrm{H}$ NMR (Figure S1) and EA analyses (Table S3), successful hydrolysis of the amide functions was achieved for all binary copolymers including those with pendant imidazole moieties (entries 3-5, Table 3). In addition, the drastic decrease or disappearance of the carbonyl peak at $1700 \mathrm{~cm}^{-1}$ in the infrared spectra confirmed the efficient removal of the acetamide groups in the copolymers (Figure S5). Finally, the hydrolysis procedure involving $\mathrm{HCl} 6 \mathrm{~N}$ was applied to a ternary copolymer composed of similar molar fractions of NVA, NMVA and VIm (entry 6, Table 3), producing the desired $\mathrm{P}(\mathrm{NVAm}$-stat-NMVAm-stat-VIm) multiamine copolymer.

\section{Conclusion}

The two-step strategy developed here is particularly efficient for the preparation of pendant primary, secondary amines and/or imidazole bearing copolymers with predictable composition. The latter consists in the synthesis of $N$-vinylamides and $N$-vinylimidazole-containing copolymers followed by the hydrolysis of the pendant amide moieties. The free radical copolymerization of NVA, NMVA and/or VIm was studied first and the reactivity ratios of each binary system were measured by different methods, i.e. Fineman-Ross, the regular and extended Kelen-Tudos as well as the nonlinear approach. The four calculation methods provided similar reactivity ratios, which demonstrates the accuracy of the values. Although these reactivity ratios were determined with a free radical polymerization system, these data could also be very helpful for future investigations aiming at controlling the molecular parameters and architectures of the copolymers through living/controlled radical copolymerization mechanisms. The reactivity of the monomers was determined to be in the following order: VIm > NVA > NMVA. The composition drift phenomenon was also evaluated by the Skeist's model and was found significant for all copolymerizations when high monomer conversions were targeted. However, stopping the copolymerizations at moderate monomer conversion allowed preventing a strong inhomogeneity of the chemical composition over the chain distribution. The compositions of the P(NVA-stat-NMVA-stat-VIm) terpolymers were also successfully predicted with the AlfreyGoldfinger equations on the basis of the reactivity ratios determined from the binary copolymerizations. A good agreement was found between the predicted and the experimentally determined values. Thanks to this copolymerization in-depth study, a series of statistical binary and ternary copolymers with known compositions were prepared and treated with acid in order to generate the pendant primary and/or secondary amines derivatives. Optimization of the hydrolysis conditions notably allowed reaching high levels of conversion of the $N$-methyl acetamide moieties within manageable reaction times, which is essential for the design of copolymers containing high densities of pendant secondary amines. Overall, we demonstrated the efficiency of this method for the preparation of statistical amine-rich copolymers containing primary and secondary as well as imidazole functions in adjustable proportions.

These achievements represent a great opportunity to finely tune the properties of PVAms, in order to improve their applications and to develop new ones. Indeed, the introduction of secondary amines and/or imidazole group along the PVAm backbone will undoubtedly modify its degree of ionization depending on $\mathrm{pH}$, its metal/macromolecule complexation ability, and surface adhesion 
capacity, which are key properties of this class of materials. For example, micro- and nanohydrogels ${ }^{49}$ formed with such unrivaled polyamines should exhibit unique $\mathrm{pH}$-responsiveness ${ }^{50,51}$ that could be tuned accurately for drug release applications by adjusting the content of each pendant amino group. In addition, the presence of various amines and imidazole with specific metal binding capacities along the same polymer backbone opens new opportunities notably in the field of water purification. ${ }^{52,53}$ Previous studies also evidenced the antibacterial properties of PVAm derivatives ${ }^{54}$ and polyvinylimidazole ${ }^{55}$, as well as the dependence of the antimicrobial efficiency on the charge density and hydrophobicity. ${ }^{54}$ Finally, these novel polyamines might also outperform primary PVAm in applications like paper strengthening or coating applications ${ }^{9-11}$. In other words, much can be expected from this poly(vinylamine) based synthetic platform.

\section{Acknowledgements}

The authors are grateful to the European Community in the frame of the Erasmus Mundus International doctoral school IDSFunMat, the UPMC University, the Belgian National Funds for Scientific Research (F.R.S.-FNRS) and the Belgian Science Policy (PAI VII05) for their financial support. A.D. is Research Associate of the FRS- FNRS. The authors also thank P. De Tullio and C. Troufflard (UPMC) for skillful NMR assistance.

\section{Notes and references}

a Center for Education and Research on Macromolecules (CERM), Department of Chemistry, University of Liege (ULg), Sart-Tilman, Allée de la Chimie 3, Bat. B6a, B-4000 Liège, Belgium. adebuigne@ulg.ac.be

${ }^{\mathrm{b}}$ Sorbonne Universités, UPMC Univ Paris 06, CNRS, Institut Parisien de Chimie Moléculaire, Team « Chimie des Polymères », 4 Place Jussieu, F75005 Paris, France. jutta.rieger@upmc.fr

$\dagger$ Electronic Supplementary Information (ESI) available: additional copolymerization data, ${ }^{1} \mathrm{H}$ NMR and HSQC spectra, size exclusion chromatography and infra-red analyses. See DOI: 10.1039/b000000x/

1 R. K. Pinschmidt, J. Polym. Sci. Part A Polym. Chem., 2010, 48, 2257-2283.

2 S. Kobayashi, K. Do Suh and Y. Shirokura, Macromolecules, 1989, 22, 2363-2366.

3 A. Toutianoush, A. El-Hashani, J. Schnepf and B. Tieke, Appl. Surf. Sci., 2005, 246, 430-436.

4 A. Katchalsky, J. Mazur and P. Spitnik, J. Polym. Sci., 1957, 23, 513-532.

5 R. Merindol, S. Diabang, O. Felix, T. Roland, C. Gauthier and G. Decher, ACS Nano, 2015, 9, 1127-1136.

6 R. Pelton, Langmuir, 2014, 30, 15373-15382.

7 C. Geffroy, M. . Labeau, K. Wong, B. Cabane and M. . Cohen Stuart, Colloids Surfaces A Physicochem. Eng. Asp., 2000, 172, 47-56.

8 Q. Chen and L. Zhu, in Applied Mechanics and Materials, 2012, vol. 130-134, pp. 1507-1510.

9 S. Wang, M. Wang and F. Chen, BioResources, 2014, 10, 750-759.

10 H. Kroener, Wochenblatt fuer Pap., 2009, 137, 172-175.

11 R. Pelton and J. Hong, Tappi J., 2002, 1, 21-25.

12 S. Yuan, Z. Wang, Z. Qiao, M. Wang, J. Wang and S. Wang, J. Memb. Sci., 2011, 378, 425-437.

13 P. Li, Z. Wang, Y. Liu, S. Zhao, J. Wang and S. Wang, J. Memb. Sci., 2015, 476, 243-255.

14 B. M. Novak and J. T. Cafmeyer, J. Amer. Chem. Soc., 2001, 123, 11083-11084.
15 M. Mullier and G. Smets, J. Polym. Sci., 1957, 23, 915-930.

16 A. El Achari, X. Coqueret, A. Lablache-Combier and C. Loucheux, Die Makromol. Chemie, 1993, 194, 1879-1891.

17 L. Gu, S. Zhu and A. N. Hrymak, J. Appl. Polym. Sci., 2002, 86, 3412-3419.

18 E. Witek, M. Pazdro and E. Bortel, J. Macromol. Sci. Part A, 2007, 44, 503-507.

19 M. Zhu, E. B. Radcliffe, D. W. Ragsdale, I. V. MacRae and M. W. Seeley, Agric. For. Meteorol., 2006, 138, 192-202.

20 M. Akashi, S. Nakano and A. Kishida, J. Polym. Sci. Part A Polym. Chem., 1996, 34, 301-303.

21 M. Akashi, S. Saihata, E. Yashima, S. Sugita and K. Marumo, J. Polym. Sci. Part A Polym. Chem., 1993, 31, 1153-1160.

22 Y. Maki, H. Mori and T. Endo, Macromol. Chem. Phys., 2007, 208, 2589-2599.

23 A. Sekiya, M. Tamura, H. Ishida and M. Watanabe, Chem. Lett., 1988, 1223-1224.

24 X. Chen, Y. Wang and R. Pelton, Langmuir, 2005, 21, 11673-11677.

25 J. Zhu and R. E. Marchant, Biomacromolecules, 2006, 7, 1036-1041.

26 H. Mokhtari, R. Pelton and L. Jin, J. Colloid Interface Sci., 2014, 413, 86-91.

27 S. Rupp, M. von Schickfus, S. Hunklinger, H. Eipel, A. Priebe, D. Enders and A. Pucci, Sensors Actuators, B Chem., 2008, 134, 225229.

28 A. Scorilas, A. Bjartell, H. Lilja, C. Moller and E. P. Diamandis, Clin. Chem., 2000, 46, 1450-5.

29 C. G. Overberger and S. Kikyotani, J. Polym. Sci. Polym. Chem. Ed., 1983, 21, 525-540.

30 R. K. Pinschmidt, L. A. Wasowski, G. G. Orphanides and K. Yacoub, Prog. Org. Coatings, 1996, 27, 209-218.

31 R. K. Pinschmidt, W. L. Renz, W. E. Carroll, K. Yacoub, J. Drescher, A. F. Nordquist and N. Chen, J. Macromol. Sci. Part A, 1997, 34, 1885-1905.

32 Y. Yuan, F. Gong, Y. Cao, W. Chen, D. Cheng and X. Shuai, J. Biomed. Nanotechnol., 2015, 11, 668-679.

33 C. L. Gebhart and A. V. Kabanov, J. Control. Release, 2001, 73, 401-416.

34 O. V. Okatova, I. I. Gavrilova, N. N. Ul'yanova, E. F. Panarin and G. M. Pavlov, Russ. J. Appl. Chem., 2012, 85, 1239-1246.

35 H. C. W. M. Buys, F. F. Vercauteren, A. van Elven and A. H. A. Tinnemans, Recl. des Trav. Chim. des Pays-Bas, 2010, 108, 123-127.

36 G. Odian, Principles of Polymerization, 4th Edition, 2004.

37 M. Fineman and S. D. Ross, J. Polym. Sci., 1950, 5, 259-262.

38 T. Kelen and F. Tudos, J. Macromol. Sci. Part A - Chem., 1975, 9, 127.

39 A. Wamsley, B. Jasti, P. Phiasivongsa and X. Li, J. Polym. Sci. Part A Polym. Chem., 2004, 42, 317-325.

40 J. M. Ting, T. S. Navale, F. S. Bates and T. M. Reineke, ACS Macro Lett., 2013, 2, 770-774.

41 I. Skeist, J. Am. Chem. Soc., 1946, 68, 1781-1784.

42 T. Alfrey and G. Goldfinger, J. Chem. Phys., 1944, 12, 322.

43 T. Kelen, F. Tudos, B. Turcsányi and J. P. Kennedy, J. Polym. Sci. Polym. Chem. Ed., 1977, 15, 3047-3074.

44 A. Debuigne, A. N. Morin, A. Kermagoret, Y. Piette, C. Detrembleur, C. Jerome and R. Poli, Chem. - Eur. J., 2012, 18, 12834-12844. 
45 K. Nakabayashi and H. Mori, Eur. Polym. J., 2013, 49, 2808-2838.

46 A. Kermagoret, C.-A. Fustin, M. Bourguignon, C. Detrembleur, C. Jerome and A. Debuigne, Polym. Chem., 2013, 4, 2575-2583.

47 A. Kermagoret, K. Mathieu, J.-M. Thomassin, C.-A. Fustin, R. Duchene, C. Jerome, C. Detrembleur and A. Debuigne, Polym. Chem., 2014, 5, 6534-6544.

48 M. Akashi, E. Yashima, T. Yamashita, N. Miyauchi, S. Sugita and K. Marumo, J. Polym. Sci. Part A Polym. Chem., 1990, 28, 3487-3497.

49 B. H. Tan, J. P. K. Tan and K. C. Tam, in Hydrogel Micro and Nanoparticles, Wiley-VCH Verlag GmbH \& Co. KGaA, Weinheim, Germany, 2012, pp. 81-115.

50 K. Yamamoto, T. Serizawa, Y. Muraoka and M. Akashi, Macromolecules, 2001, 34, 8014-8020.

51 L. Jin, Q. Sun, Q. Xu and Y. Xu, Bioresour. Technol., 2015, 197, 348-355.

52 A. K. Kushwaha, N. Gupta and M. C. Chattopadhyaya, Desalin. Water Treat., 2014, 1-13.

53 R. Wang, J. Men and B. Gao, CLEAN - Soil, Air, Water, 2012, 40, 278-284.

54 E.-H. Westman, M. Ek, L.-E. Enarsson and L. Wågberg, Biomacromolecules, 2009, 10, 1478-1483.

55 H. El-Hamshary, M. M. G. Fouda, M. Moydeen, M. H. El-Newehy, S. S. Al-Deyab and A. Abdel-Megeed, Int. J. Biol. Macromol., 2015, 72, 1466-1472. 